\title{
Selective Two-Photon Absorptive Resonance Femtosecond- Laser Electronic-Excitation Tagging (STARFLEET) Velocimetry in Flow and Combustion Diagnostics
}

\author{
Naibo Jiang, ${ }^{1}$ Benjamin R. Halls, ${ }^{2}$ Hans U. Stauffer, ${ }^{2}$ and Sukesh Roy ${ }^{3}$ \\ Spectral Energies, LLC, 5100 Springfield St., Dayton, OH 45431 \\ Paul M. Danehy ${ }^{3}$ \\ NASA Langley Research Center, Hampton, VA23681 \\ and \\ James R. Gord ${ }^{4}$ \\ Aerospace Systems Directorate, Air Force Research Laboratory, Wright-Patterson Air Force Base, OH 45433
}

\begin{abstract}
Selective Two-Photon Absorptive Resonance Femtosecond-Laser Electronic-Excitation Tagging (STARFLEET), a non-seeded ultrafast-laser-based velocimetry technique, is demonstrated in reactive and non-reactive flows. STARFLEET is pumped via a two-photon resonance in $\mathrm{N}_{2}$ using $202.25-\mathrm{nm}$ 100-fs light. STARFLEET greatly reduces the per-pulse energy required (30 $\mu \mathrm{J} /$ pulse) to generate the signature FLEET emission compared to the conventional FLEET technique (1.1 $\mathrm{mJ} /$ pulse). This reduction in laser energy results in less energy deposited in the flow, which allows for reduced flow perturbations (reactive and nonreactive), increased thermometric accuracy, and less severe damage to materials. Velocity measurements conducted in a free jet of $\mathrm{N}_{2}$ and in a premixed flame show good agreement with theoretical velocities and further demonstrate the significantly less-intrusive nature of STARFLEET.
\end{abstract}

\section{Introduction}

$I_{\mathrm{c}}^{\mathrm{N}}$ $\mathrm{N}$ recent years, laser-based velocity-measurement techniques have been widely developed and applied in the areas of flow and combustion diagnostics. Accurate velocity-field measurements aid the determination of other flow-transport-related quantities such as vorticity and shear stress [1-3]. These measurements are also instrumental in the evaluation of numerical models. In most velocimetry techniques, such as Particle Imaging Velocimetry (PIV) [4, 5], Molecular Tagging Velocimetry (MTV) [6, 7], Planar Doppler Velocimetry (PDV) [3, 8], and Laser Doppler Velocimetry (LDV) [9], seed particles or molecules are added into the flow and tracked. By measuring the displacements of the seed material within a certain time delay, the 1-D or 2-D velocity field can be determined. However, the addition of seed particles or molecules either directly perturbs the flow or changes the flow-field characteristics. These seeded tracers can also be harmful to sensitive ground test facilities. Raman Excitation LaserInduced Electronic Fluorescence (RELIEF) [10,11], which is based on the fluorescence of the oxygen molecule, is one of a few techniques that does not require seeding. However, RELIEF is complicated by Raman excitation and Planar Laser-Induced Fluorescence (PLIF) occurring simultaneously. Rayleigh-scattering velocimetry [12], laser-induced thermal acoustics (LITA) [13] and air photolysis and recombination tracking (APART) [14] are also unseeded velocimetry techniques, each having its own limitations in terms of dynamic range, spatial resolution and complexity. Another non-seeded velocimetry technique, Femtosecond-Laser Electronic-Excitation Tagging (FLEET), was first described in 2011 [15] and subsequently applied toward flow-velocimetry applications [16, 17]. FLEET is a nitrogen-fluorescence-based technique that requires only a femtosecond (fs)duration laser and a camera. Nitrogen molecules are photodissociated by a fs laser pulse to form significant populations of $\left.\mathrm{N}^{4} \mathrm{~S}\right)$

\footnotetext{
${ }^{1}$ Research Scientist, AIAA Senior Member

${ }^{2}$ Research Scientist, AIAA Member

${ }^{3}$ Research Scientist, AIAA Associate Fellow

${ }^{4}$ Principal Research Scientist, AIAA Fellow
} 
atoms [15], which undergo three-body recombination to form metastable, electronically excited nitrogen $\mathrm{N}_{2}\left({ }^{5} \Sigma{ }_{g}^{+}\right)$and, subsequently, a vibrationally excited nitrogen $B$ state. Figure 1 shows the $\mathrm{N}_{2}$ energy level diagram. The flow-velocity information can be obtained by measuring fluorescence from the first positive band of $\mathrm{N}_{2}\left(B^{3} \Pi_{\mathrm{g}} \rightarrow A^{3} \Sigma_{u}^{+}\right)$, which has a relatively long lifetime $(\sim 20 \mu$ s). If an $\sim 800-n m$ fs-duration laser pulse is used, the photodissociation step requires absorption of seven or more photons to overcome the 9.8-eV dissociation energy of $\mathrm{N}_{2}$. Typical FLEET measurements, therefore, require the use of a sharply focused 100-fs laser beam to induce multi-photon absorption; such conditions tend to photodissociate many other species within the medium, which results in local heating and, in some cases, significant alteration of the local chemistry. For example, typical FLEET studies employ 1-3 mJ of laser pulse energy focused to a small beam diameter $(\sim 100 \mu m)$, which can lead to significant local temperature increases [18].

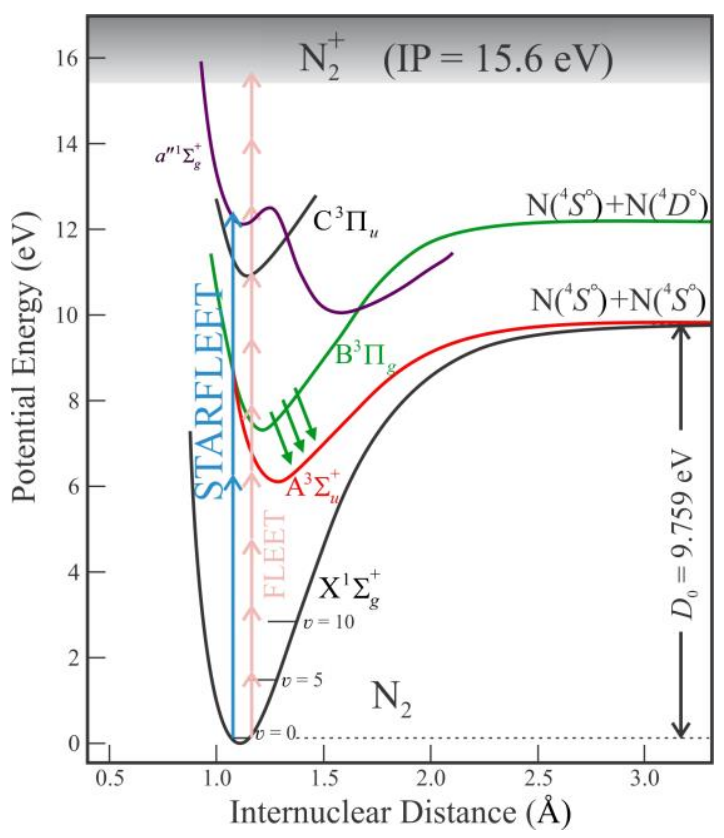

Fig. 1. $\mathrm{N}_{2}$ energy-level diagram depicting FLEET and STARFLEET excitation and fluorescence schemes.

In some cases, such as in hypersonic flows and combustion, it is critical that the high-intensity probe-laser pulses do not perturb the flow fields or chemistry. For velocimetry applications of FLEET in large ground-test facilities [19], high-energy fs laser pulses could also potentially damage the expensive wind tunnel model. Edwards et al. [18] measured the effects of FLEET laser heating by observing spectrally resolved fluorescence from the second positive band $\left(C^{3} \Pi_{u}-B^{3} \Pi_{g}\right)$ of nitrogen. The local flow temperature was determined by fitting the second positive rotational envelope around $358 \mathrm{~nm}$. Based on the assumption that rotational and translational temperatures equilibrate within 10 nanoseconds at atmospheric pressure, Edwards et al. observed that flow temperatures increased by as much as $100 \mathrm{~K}$ as a result of additional energy deposition into the flow field. Ryabtsev et al. [20] also reported that fs-laser photodissociation and ionization of the medium deposit a non-negligible amount of heat that creates wake vortices and turbulence in the flow field. To avoid the introduction of additional laser heating or fluid perturbation, it is desirable to use low laser pulse energies - well below the $\sim \mathrm{mJ} /$ pulse energies used in typical FLEET experiments - that can still produce sufficient FLEET signals. The Selective Two-photon Absorptive Resonance FLEET (STARFLEET) [21] approach presented here has been developed to address this concern. The STARFLEET approach, also depicted in Fig. 1, is designed to exploit the resonant excitation of the $\mathrm{N}_{2} a^{\prime \prime 1} \Sigma_{g}^{+} \leftarrow X^{1} \Sigma_{g}^{+}$transition [22] via two-photon absorption at $\sim 202.25 \mathrm{~nm}$. Predissociation in the $a^{\prime \prime 1} \Sigma_{g}^{+}$manifold or subsequent absorption of a third photon from this electronic state is expected to dissociate the $\mathrm{N}_{2}$ molecule efficiently, resulting in a pool of $\mathrm{N}$ atoms. The STARFLEET approach has the same capabilities as FLEET in obtaining temperature and velocity by simultaneously recording the electronically excited $\mathrm{N}_{2}$ emission spectra. The significant advantage is that two-photon absorption technique requires only several microjoules $(\mu \mathrm{J})$ of fs $\mathrm{UV}$ radiation to initiate the process leading to the long-lived emission. 


\section{Experiments}

The STARFLEET absorption wavelength at $\sim 202.25 \mathrm{~nm}$ is produced by fourth-harmonic generation (FHG) [23] of the 810$\mathrm{nm}(\sim 6 \mathrm{~mJ} /$ pulse) emission of a 1-kHz Coherent Legend Elite Duo Ti:sapphire laser. For all of the flowing-gas STARFLEET studies, the excitation laser power is $\sim 30 \mu \mathrm{J} /$ pulse with $\sim 5 \mathrm{~mm}$ beam diameter and focused at the measurement volume using a 250-mm focal-length lens. The gas is flowed at room temperature and atmospheric pressure. A PI-MAX IV ICCD camera is used to record the emission signals emanating from the measurement volume. The ICCD camera was coupled with a 50-mm Nikon lens and was operated with $70 \%$ gain. The distance between the camera lens and the measurement volume is $~ 100 \mathrm{~mm}$. Decay-lifetime measurements of emission signals were carried out using four different spectral filtering configurations as follows: (1) unfiltered; (2) 550-nm long-pass filter; (3) 520-700-nm colored-glass filter; and (4) 325-385-nm band-pass filter. Here, configurations 2 and 3 are used to isolate the visible first positive band range observed in typical prior FLEET measurements, whereas configuration 4 isolates the $\mathrm{N}_{2}$ second positive fluorescence band range. In examples where the rotational temperature of the emission band is determined, experimental spectra were fit to simulated spectra using a commercially available software package (SPECAIR).

\section{Results and Discussions}

Figure 2 shows the STARFLEET signal intensity, obtained using spectral filter configuration 3 at $3 \mu$ s delay and $1 \mu$ sate width, at various excitation-pulse wavelengths in both air and pure $\mathrm{N}_{2}$. Under both conditions, resonance-enhancement effects associated with the excitation pulse are clearly observed in the $\mathrm{N}_{2}$ fluorescence-excitation spectrum. The background signal level of $\sim 500$ counts, observed in both $\mathrm{N}_{2}$ and air, has been removed and the peak intensities have been normalized to the maximum signal in the data shown in Fig. 2; the maximum peak intensity is $\sim 21,000$ counts for $\mathrm{N}_{2}$ and 5,000 for air. Therefore, the resonant excitation produces almost a 40-fold enhancement of fluorescence signal than is observed under off-resonance conditions in pure $\mathrm{N}_{2}$ and a 10-fold increase in signal over background in air. These results clearly demonstrate the resonance effects associated with the observed $\mathrm{N}_{2}$ fluorescence. Note that the focal conditions used in the experiment are observed to have a notable effect on the degree of resonance enhancement over background non-resonant fluorescence signal. For example, when a 100-mm focallength lens is used, only a 3 -fold resonance enhancement is observed.

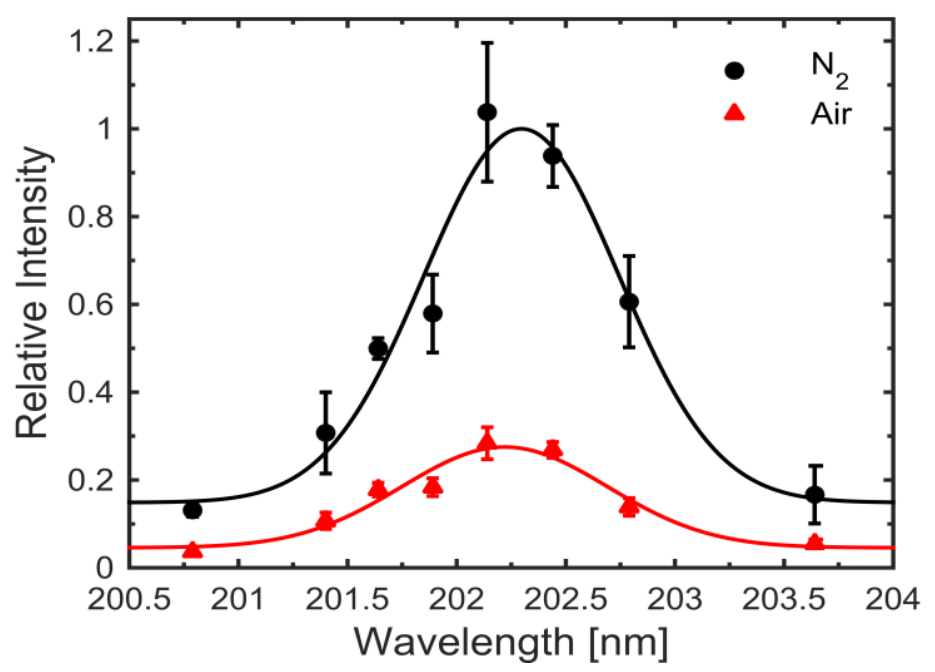

Fig. 2. STARFLEET fluorescence-excitation spectrum depicting wavelength dependence of observed signal with $2 \sigma$ uncertainties. The ratio of resonant fluorescence signal to off-resonant signal is 8:1 in pure $\mathrm{N}_{2}$ and 5:1 in air. Solid curves included are Gaussian fits to guide the eye.

Figure 3 shows a comparison of STARFLEET signal lifetimes observed in both pure $\mathrm{N}_{2}$ and air. In Fig. 3(a) at similar laser power and camera delay times, the STARFLEET signal in pure $\mathrm{N}_{2}$ is about 10 times stronger than that in air. Similar phenomena are also observed in the standard FLEET experiments. Figure 3(a) also illustrates a shorter emission lifetime $(\sim 5 \mu \mathrm{s})$ at room temperature and atmospheric pressure with STARFLEET as compared to standard FLEET, which usually exhibits $\sim 20 \mu$ s decay times. The initial signal-intensity ratio between $\mathrm{N}_{2}$ and air is $\sim 20$, using the same energy, which is consistent with previous reported FLEET results [16]. The difference in signal intensites is likely the result of an increase in the rate of fluorescence 
quenching in the presence of oxygen. In Fig. 3(b), several STARFLEET intensity versus time traces using various filter conditions associated with different emission bands are shown. For all observed emission bands, the STARFLEET signal generally exhibits a fast decay ( 100 ns lifetime) and a slow decay ( 5 $\mu$ s lifetime). The fast-decay signal is attributed to shortlived fluorescence from nitrogen molecules that undergo intersystem crossing into the triplet manifold following initial excitation. The slow-decay lifetimes with various filter conditions appear to be very similar, which is consistent with the assumption of longlived recombination of $\mathrm{N}$-atom as the rate limiting step. As discussed earlier, after dissociation occurs, the FLEET or STARFLEET signal will depend on the $\mathrm{N}$-atom recombination that is related to the rate of collision. The slow-decay signal also implies that the laser beam at a resonant absorption wavelength efficiently produces the pool of dissociated $\mathrm{N}$ atoms. Here the nitrogen second positive band also shows a long-decay lifetime which is different from the standard FLEET, which probably implies that the $\mathrm{N}$-atom recombination scheme is different for FLEET and STARFLEET. Further experiments are needed to clarify this phenomenon.
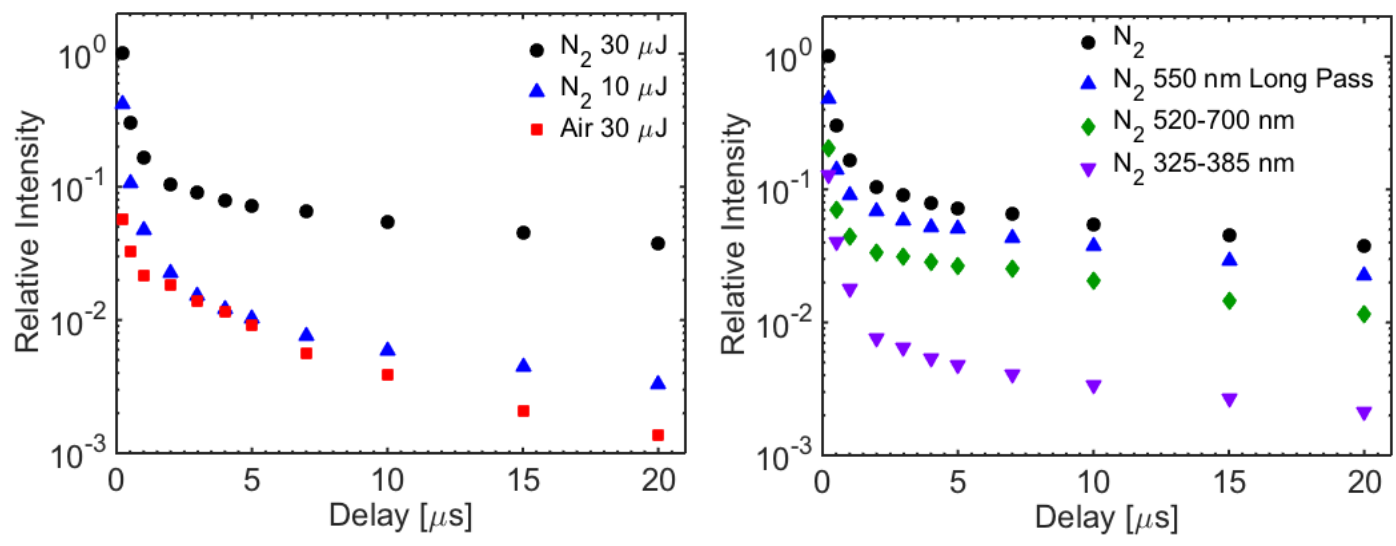

Fig. 3. (a) STARFLEET lifetime in $\mathrm{N}_{2}$ and air by a 202.25-nm fs pulse excitation. (b) Lifetime of various bands of STARFLEET signal in $\mathrm{N}_{2}$. All of the cases show a fast decay of $\sim 100 \mathrm{~ns}$ and a slow decay of $\sim 5 \mu \mathrm{s}$.

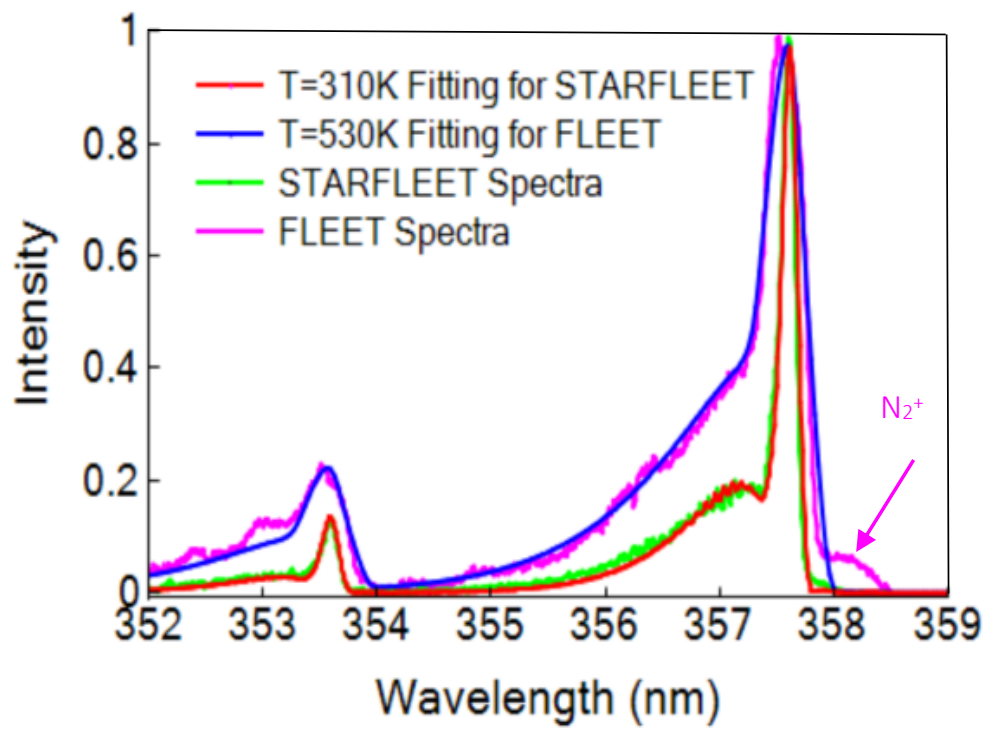

Fig. 4. Comparison of observed $\mathrm{N}_{2}$ second positive band spectra for STARFLEET and FLEET. Experimental results shown as symbols. Best-fit rotational temperatures (solid curves) are found to be 200 K higher for FLEET than for STARFLEET. 
The most important advantage of STARFLEET is its less invasive nature relative to FLEET by virtue of reduced and selective energy deposition. With a $5-\mu$ s delay time, typical for velocimetry measurements, the same fluorescence intensity is produced for STARFLEET and FLEET with energies of $30 \mu \mathrm{J} /$ pulse and $1.1 \mathrm{~mJ} /$ pulse, respectively; thus, a factor of 30 times power reduction is achieved through the use of the resonance-based approach in STARFLEET as compared to conventional FLEET. Figure 4 shows a comparison of STARFLEET and FLEET spectra associated with the second positive (0-1) band of $\mathrm{N}_{2}$ near $358 \mathrm{~nm}$, obtained in a $\sim 300 \mathrm{~K}$ nitrogen flow. With normalized signal intensities, the standard FLEET signal exhibits more pronounced rotational band envelope, a broader band head, and more-pronounced presence of a $\mathrm{N}_{2}{ }^{+}$peak around $358.3 \mathrm{~nm}$ [19] than STARFLEET. The $\mathrm{N}_{2}{ }^{+}$peak for STARFLEET is below the detection limit, but the presence of the FLEET spectrum implies that there is significantly more ionization of nitrogen molecules with FLEET than that of STARFLEET. In order to quantify the degree of localized heating induced by both the FLEET and STARFLEET approaches, fitting of the rotational temperature using SPECAIR software are also shown in Figure 4. Here, a wider slit was used in experiment for the FLEET measurement to capture the spectrum with a good signal-to-noise ratio. However, the reduced resolution in fitting was accounted for in the simulated fits. A best-fit rotational temperature of $\sim 530 \mathrm{~K}$ was obtained for the FLEET results; whereas a temperature of $\sim 310 \mathrm{~K}$ is optimal to match the STARFLEET results.

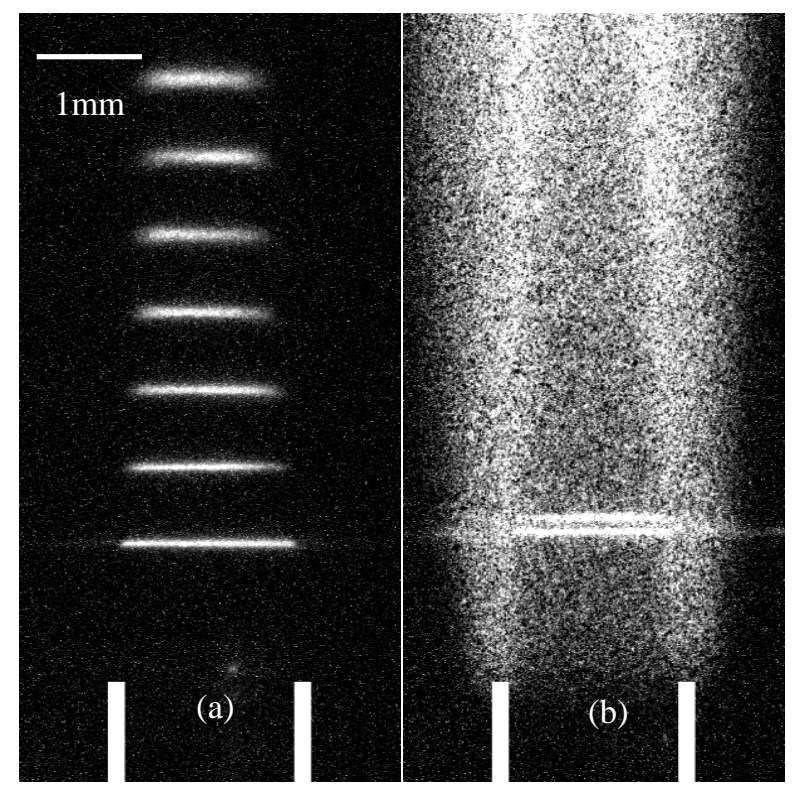

Fig. 5. STARFLEET Velocimetry examples in (a) $170 \mathrm{~m} / \mathrm{s} \mathrm{N}_{2}$ jet flow and (b) $27 \mathrm{~m} / \mathrm{s}$ premixed flame.

Successful demonstration of velocimetry using the STARFLEET approach has been conducted in both a $\mathrm{N}_{2}$ free jet and a premixed $\mathrm{H}_{2}, \mathrm{O}_{2}$, and $\mathrm{N}_{2}$ flame using 202-nm STARFLEET, as shown in Fig. 5(a) and 5(b), respectively. Figure 5(a) shows cumulative shots of the free $\mathrm{N}_{2}$ jet (dia. $2.5 \mathrm{~mm}$ ) with a flow rate of 50 standard liters per minute (slpm). Measurements were conducted every $5 \mu \mathrm{s}$, starting at $0.2 \mu \mathrm{s}$ after the laser pulse up to $30.2 \mu \mathrm{s}$. The measured core flow velocity was $184 \mathrm{~m} / \mathrm{s}$ as compared to the simple estimated average jet core velocity of $\sim 170 \mathrm{~m} / \mathrm{s}$ based on the flow rate and jet diameter. The STARFLEET signal beam appears to be wider for the longer delay time because of turbulence and diffusion. This measurement was obtained with a PIMAX-IV ICCD camera. In Fig. 5(b), the measurements were conducted within a premixed $\mathrm{H}_{2} / \mathrm{O}_{2} / \mathrm{N}_{2}$ flame. Similar cumulative exposure was obtained for the same jet. Flow rates were $2 \mathrm{slpm}$ for $\mathrm{H}_{2}, 1 \mathrm{slpm}$ for $\mathrm{O}_{2}$, and 5 slpm for $\mathrm{N}_{2}$. The bright background in Fig. 5(b) results from the flame chemiluminescence. Measurements were obtained at $0.2 \mu \mathrm{s}$ and 5.2 $\mu$ s after the laser pulse. The measured velocity was $32 \mathrm{~m} / \mathrm{s}$ as compared to the calculated bulk velocity of $\sim 27 \mathrm{~m} / \mathrm{s}$. The signal is stronger in the core region of the flame, which is unburned fuel, $\mathrm{O}_{2}$, and $\mathrm{N}_{2}$ before the flame front. After the flame front, the temperature increases significantly, with a corresponding reduction in nitrogen density, and the gas composition is also altered; therefore, the observed signal is much weaker in this region. The jet and flame measurements using STARFLEET show that not only can the technique reproduce FLEET results but also it can transition the technique to more difficult and complex environments. 
Finally, the reduced invasiveness of STARFLEET has been demonstrated in material-damage studies, since low-velocity measurements are often carried out in close proximity to expensive aerodynamic models, where minimization of damage is a necessity. Two materials - aluminum and stainless steel—were tested for laser damage with FLEET and STARFLEET. Both materials had unpolished surfaces and were 1-mm thick. Again, $30 \mu \mathrm{J} /$ pulse of laser power for STARFLEET and $\sim 1.1 \mathrm{~mJ} / \mathrm{pulse}$ for FLEET were applied in this test to match the experimental configurations expected to be necessary in velocimetry measurements. A 100-mm lens was used to focus the laser beam. Each plate was fixed at the focal point for about $10 \mathrm{~min}$. For both the aluminum and stainless-steel plates pumped with 202 -nm light, a very small dark burn point appeared on the plate surface, which is difficult to distinguish. However, with 1.1-mJ/pulse pump power at $810 \mathrm{~nm}$, a large burn mark was present on the aluminum surface, and the laser drilled through the 1-mm stainless-steel plate in $<30 \mathrm{~s}$.

The two-photon-resonance-based STARFLEET technique has been successfully demonstrated with improvements over traditional FLEET. The new velocimetry technique takes advantage of resonance-enhanced multi-photon absorption processes of $\mathrm{N}_{2}$ near the fourth harmonic of an amplified fs Ti:sapphire laser; therefore, significantly reduced power requirements are achieved. Both spectrum and material-damage comparisons demonstrate the less-invasive nature of the STARFLEET approach. Measurements conducted on the $\mathrm{N}_{2}$ free jet and premixed flames show excellent agreement. The STARFLEET approach significantly reduces the photon energies needed for fs-pulse-based $\mathrm{N}_{2}$ velocimetry approaches and minimizes the invasive nature of high-peak-power fs-duration pulses in the conventional FLEET method. Such innovative methods will represent a critical step forward in applying optical diagnostics with reduced energy deposition and reduced risk of damage to models in wind-tunnel facilities and will allow its application in high-enthalpy flows involving chemical reactions. The $\mathrm{N}_{2}$ emission intensity is linearly proportional to the incident UV pulse intensity with decay lifetimes that are shorter than those observed in FLEET experiments. This indicates that further exploration of multiple-beam techniques will enhance the overall performance of the velocimetry technique. We plan to continue to explore this by looking at possible enhancement of two-color excitation. The FHG setup used for generation of 202.25-nm STARFLEET excitation involves incremental generation of second and third

harmonics of the $\sim 810$-nm fundamental pulse; therefore, subsequent excitation of the two-photon-excited $a^{\prime \prime 1} \Sigma_{g}^{+}$state using 810-nm, or 202.25-nm light can be used to maximize the pool of $\mathrm{N}$ atoms necessary to initiate FLEET emission.

\section{Acknowledgments}

We thank Drs. Waruna Kulatilaka, Jake Schmidt, and Paul Hsu, for their assistance and many useful discussions associated with this work. This material is based upon work supported by Air Force Office of Scientific Research award Nos. 15RQCOR202 and 14RQ06COR, and NASA SBIR Contract Nos. NNX14CL74P \& NNX15CL24C. Approved for public release: distribution unlimited (\#88ABW-2016-2172).

\section{References}

1. R.J. Andrian, "Twenty years of paricle image velocimetry", Experiments in Fluids, 39, 159-169, (2005).

2. W.R. Lempert, M. Boehm, N. Jiang, S. Gimelsein and D. Levin, "Comparison of molecular tagging velocimetry data and direct simulation Monte Carlo simulations in supersonic micro jet flows", Experiments in Fluids, 34, 403-411, (2003).

3. B.S. Thurow, N. Jiang, J.H. Kim, W.R. Lempert and M. Samimy, "Issues with measurements of the convective velocity of large-scale structures in the compressible shear layer of a free jet", Physics of Fluids, 20, 066101, (2008).

4. J. Westerweel, G.E. Elsinga and R.J. Adrian, "Particle image velocimetry for complex and turbulent flows", Annual Review of Fluid Mechanics 45, 409-436 (2013).

5. P.S. Hsu, S. Roy, N. Jiang and J.R. Gord, "Large-aperture, tapered fiber-coupled, 10-kHz particle-image velocimetry", Optics Express 21, 3617-3626 (2013).

6. C.P. Gendrich, M.M. Koochesfahani and D.G. Nocera, "Molecular tagging velocimetry and other novel applications of a new phosphorescent supramolecule", Experiments in Fluids 23, 361-372 (1997).

7. W.R. Lempert, N. Jiang, S. Sethuram and M. Samimy, "Molecular tagging velocimetry measurements in supersonic microjets", AIAA Journal, 40, 1065-1070 (2002).

8. R.L. McKenzie, "Measurement capabilities of planar Doppler velocimetry using pulsed lasers", Applied Optics 35, 948964, (1996).

9. J.W. Foreman, E.W. George and R.D. Lewis, "Measurement of localized flow velocities in gases with a laser Doppler flowmeter", Applied Physics Letters 7, 77 (1965).

10. R. Miles, C. Cohen, P. Howard, S. Huang, E. Markovitz and G.Russell, "Velocity measurements by vibrational tagging and fluorescent probing of oxygen", Optics Letters 12, 861-863 (1987).

11. W.R. Lempert, Y. Zuzeek, M. Uddi, K. Frederickson, N. Jiang, S. Roy, T. Meyer, S. Gogineni and J.R. Gord, "RELIEF velocimetry using picosecond tagging and Nd:YAG-based interrogation”, AIAA-2006-2970 (2006).

12. J.N. Forkey, N. D. Finkelstein, W. R. Lempert, and R. B. Miles, "Demonstration and characterization of filtered Rayleigh scattering for planar velocity measurements", AIAA journal, 34, 442-448, (1996). 
13. E.B. Cummings, "Laser-induced thermal acoustics: simple accurate gas measurements", Optics letters, 19, 1361-1363, (1994).

14. N. J. Dam, R. J. H. Klein-Douwel, N. M. Sijtsema, and J. J. ter Meulen, "Nitric oxide flow tagging in unseeded air", Optics Letters, 26, 36-38, (2001).

15. J.B. Michael, M.R. Edwards, A. Dogariu and R. B. Miles, "Femtosecond laser electronic excitation tagging for quantitative velocity imaging in air", Applied Optics 50, 5158-5162 (2011).

16. N.J. Deluka, R.B. Miles, W.D. Kulatilaka, N. Jiang and J.R. Gord, "Femtosecond laser electronic excitation tagging (FLEET) fundamental pulse energy and spectral response", AIAA-2014-2227, 30 ${ }^{\text {th }}$ AIAA Aviation Conference, Atlanta, GA, June 16-20 (2014).

17. P.M. Danehy, B.F. Bathel, N.D. Calvert, A. Dogariu and R.B. Miles, "Three component velocity and acceleration measurement using FLEET", AIAA-2014-2228, 30 th AIAA Aviation Conference, Atlanta, GA, June 16-20 (2014).

18. M.R. Edwards, A. Dogariu and R.B. Miles, "Simultaneous temperature and velocity measurement in unseeded air flows with FLEET", AIAA-2013-0043, 51 ${ }^{\text {th }}$ AIAA Aerospace Sciences Meeting, Dallas, TX, Jan. 7-10 (2013).

19. R. Burns, P.M. Danehy, S.B. Jones, B. R. Halls and N. Jiang, "Application of FLEET velocimetry in the NASA Langley 0,3-meter transonic cryogenic tunnel", AIAA-2015-2566, $31^{\text {th }}$ AIAA Aviation Conference, Dallas, TX, June 22-26 (2015).

20. A. Ryabtsev, S. Pouya, M. Koochesfahani and M. Dantus, "Votices in the wake of a femtosecond laser filament", Optics Express 22, 26098-26102 (2014).

21. N. Jiang, B.R. Halls, H.U. Stauffer, P.M. Danehy, J.R. Gord, and S. Roy, "Selective two-photon absorptive resonance femtosecond-laser electronic-excitation tagging velocimetry," Optics Letters 41, 2225-2228 (2016).

22. E.J. Salumbides, A. Khramov, and W. Ubachs, "High-resolution $2+1$ REMPI study of the $a^{\prime \prime} \Sigma_{g}^{+}$state in $\mathrm{N}_{2}$ ", Journal of Physical Chemistry A, 113, 2383-2386 (2009).

23. W.D. Kulatilaka, J.R. Gord, S. Roy, "Femtosecond two-photon LIF imaging of atomic species using a frequencyquadrupled Ti:sapphire laser", Applied Physics B, 116, 7-13, (2014). 